\title{
Physiologically based pharmacokinetic models for the transport of trichloroethylene in adipose tissue
}

\author{
R.A. Albanese*, H.T. Banks ${ }^{\dagger}$ M.V. Evans ${ }^{\ddagger}$ and L.K. Potter ${ }^{\dagger}$ \\ *Air Force Research Laboratory, Brooks Air Force Base, San Antonio, TX 78235; \\ ${ }^{\dagger}$ Center for Research in Scientific Computation, \\ North Carolina State University, Raleigh, NC 27695-8205;

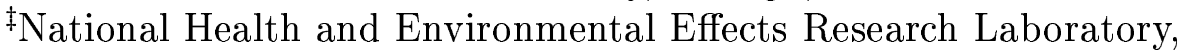 \\ U.S. Environmental Protection Agency, Research Triangle Park, NC 27711
}

\begin{abstract}
In this paper we present three physiologically based pharmacokinetic (PBPK) models for the systemic transport of trichloroethylene (TCE), with a focus on the adipose, or fat tissue. TCE is a widespread environmental contaminant, and has been shown to produce toxic effects in both animals and humans. A key characteristic of TCE is its tendency to accumulate in fat tissue, which has a major impact on the overall systemic disposition of TCE.

Here we use PBPK models to predict the kinetics of TCE through the various tissues and organs, including the adipose tissue. The first model utilizes the standard "perfusion-limited" compartmental model for the fat tissue, while the second model uses a "diffusion-limited" model to describe the transport through the adipose tissue. Both of these ODE models are based on "well-mixed" and rapid equilibrium assumptions, and do not take into account the specific and largely heterogeneous physiology of adipose tissue.

The third model we discuss is a PBPK hybrid model with an axial-dispersion type model for the adipose tissue. This PDE-based model is designed to capture key physiological heterogeneities of fat tissue, including widely varying fat cell sizes, lipid distribution, and blood flow properties. Model simulations demonstrate that this model may be well-suited to predict the experimental behavior of TCE in adipose tissue using parameter estimation techniques.
\end{abstract}

\section{Introduction}

Many environmental and occupational toxins are lipophilic in nature, and tend to accumulate in the adipose, or fat tissue of animals and humans. These compounds vary widely in their means of uptake into, disposition through, and elimination from the body, as well as in their toxic mechanisms. A key component in understanding their specific toxicities is the characterization of their kinetics. Since adipose is a storage tissue for these xenobiotics, it is important to ascertain their absorption, distribution and accumulation properties for adipose tissue.

Numerous studies have shown that a large class of lipophilic compounds accumulates in adipose tissue. This class includes organochloride pesticides such as aldrin, dieldrin, endrin, chlordane, heptachlor and DDT [2], as well as polychlorinated biphenyls [2],[36], polychlorinated dibenzo-p-dioxins and polychlorinated dibenzo-furans [15]. Moreover, a variety of other halogenated organic compounds are known to accumulate in fat tissue [2]. This tissue accumulation is related to the lipophilicity of the compounds and the low perfusion 
rate of adipose tissue, although studies in [21] and [39] suggest that the tissue distribution of lipophilic compounds is also dependent on binding competition between fat tissue and well-perfused lean tissues.

As a motivating example, we consider the solvent trichloroethylene (TCE), which is widely used in industry as a metal degreasing agent. Humans come into contact with TCE most often by inhaling TCE vapor or by drinking contaminated water. TCE is a highly lipophilic volatile organic compound, and is quickly distributed into the bloodstream. It is partially eliminated from the body through exhaled air and is rapidly metabolized in the liver. There are two major metabolic pathways for TCE: Cytochrome P450-mediated oxidation and glutathione conjugation [33]. Major metabolites include chloral hydrate, trichloroacetic acid (TCA), dichloroacetic acid (DCA), free trichloroethanol, trichloroethanol-glucuronide and $S$-(1,2-dichlorovinyl)-L-cysteine (DCVC) [20],[33], and most are eliminated in the urine [50].

Unmetabolized TCE accumulates in adipose tissue [50], but over long periods of time is not very persistent in the body because of its rapid metabolism. In a study by Bergman [8], mice were exposed to a dose of 280 $\mathrm{mg} / \mathrm{kg}$ radiolabeled TCE for ten minutes, and the tissue distribution of TCE was measured using wholebody autoradiography. Within 30 minutes, there was a large accumulation of TCE in the fat tissue, with significant levels still present after four hours and traces still visible after eight hours.

TCE and several of its metabolites are known to produce toxic effects in humans. Acute exposure to TCE impacts the central nervous system, causing symptoms such as fatigue, headaches, dizziness and drowsiness [28]. Several of its metabolites are suspected of causing toxicity in animals and/or humans, including TCA, DCA, DCVC and chloral hydrate [13],[40]. Evidence suggests that both TCA and DCA are hepatocarcinogens [13], [14] while chloral hydrate is associated with lung tumors in mice [29]. Moreover, a recent study shows that TCE may play a role in the development of kidney cancer in humans [12], and the metabolite DCVC has been linked to kidney tumors in rats [34]. TCE and some of its metabolites also have been associated with developmental toxicity in animals [43], including eye and cardiac malformations [7].

These lipophilic environmental toxins such as TCE can have both short-term and long-term adverse health effects to our population. It is important to assess the health risks these chemicals pose, so that the public can be adequately protected from dangerous compounds and the safer compounds can be used with greater confidence. In order to better determine how xenobiotics such as TCE cause toxic effects in the body, the toxicokinetics of these compounds must be understood. Physiologically based pharmacokinetic (PBPK) models are developed to describe the disposition of a given chemical throughout the body. By characterizing the kinetics of the compound, we can predict its transport inside the body and work towards gaining a better understanding of its toxic effects and mechanisms.

The standard PBPK models, which make use of "perfusion-limited" and "diffusion-limited" compartments, are designed around the assumption of rapid equilibrium and uniformity within each compartment or subcompartment. This is often a reasonable assumption in physiological modeling, but the nonhomogeneous composition, structure and circulation properties of adipose tissue suggest that this type of "well-stirred" model may not be sufficient. An axial dispersion model, such as the one derived by Roberts and Rowland [41] for hepatic uptake and elimination, is designed to account for such physiological heterogeneities.

In this paper we develop toxicokinetic models to describe the behavior of inhaled trichloroethylene as it enters, flows through, and possibly accumulates in adipose tissue. We formulate several physiologically based models, each with a different submodel for the adipose tissue component. First we derive the standard PBPK model with perfusion-limited compartments. We then modify this model by replacing the perfusion-limited adipose tissue compartment with a diffusion-limited compartment. Finally, we adapt the dispersion model of Roberts and Rowland to describe the transport of TCE through adipose tissue. This compartment is then coupled with the remaining perfusion-limited compartments to create a whole-body PBPK model. 


\section{Standard PBPK models}

\subsection{A perfusion-limited PBPK model for TCE}

Many PBPK models for lipophilic compounds include compartments for tissues such as the liver, lungs, adipose tissue, richly perfused and poorly perfused tissues. These compartments often assume a perfusionlimited model, or equivalently, a flow-limited model of disposition, meaning that the rate of uptake of the compound into the tissue is limited by the blood flow rate to the tissue rather than the rate of diffusion across the cell membranes [38]. In this case, the blood and tissue are in equilibrium and the equation for motion of a solute through the constant-volume tissue compartment is an ordinary differential equation of the form

$$
V \frac{d C}{d t}=Q\left(C_{\text {in }}-C_{\text {out }}\right)
$$

where $V$ is the volume of the tissue in liters, $C$ is the concentration of compound inside the tissue (in $\mathrm{mg} /$ liter), $Q$ is the blood flow rate to the tissue (in liters/hour), and $C_{i n}$ and $C_{\text {out }}$ are the compound concentrations entering and exiting the tissue, respectively. Examples of PBPK models for TCE and its metabolites that use perfusion-limited compartments can be found in [1], [24], [25], [30], [48].

Here we develop a standard PBPK model [38] for inhaled TCE with flow-limited compartments for the kidney, muscle tissue, adipose tissue, brain, liver, venous blood, and remaining non-fat tissue (see Figure 1). We assume uptake via inhalation, with a lung compartment subdivided into the alveolar space and lung blood subcompartments. TCE is metabolized in the liver, which is modeled with Michaelis-Menten kinetics.

In the lung, ventilation is assumed to be continuous with rate $Q_{p}$, and the vapor in the alveolar space is in rapid equilibrium with the arterial lung blood, which has cardiac output rate $Q_{c}$. Moreover, the blood/air partition coefficient is denoted by $P_{b}$.

The variables used in the lung compartment include:

$$
\begin{aligned}
C_{c} & =\text { Concentration of xenobiotic in surrounding air } \\
C_{x} & =\text { Concentration of xenobiotic in alveolar space } \\
C_{a} & =\text { Concentration of xenobiotic in arterial blood } \\
C_{v} & =\text { Concentration of xenobiotic in venous blood } \\
A_{i} & =\text { Amount of xenobiotic inhaled } \\
A_{x} & =\text { Amount of xenobiotic exhaled } \\
A_{L} & =\text { Amount of xenobiotic in lung. }
\end{aligned}
$$

In this case, the concentration $C_{x}$ in the alveolar air is related linearly to the concentration $C_{a}$ in the arterial blood:

$$
C_{x}=\frac{C_{a}}{P_{b}}
$$

The rate of inhalation of xenobiotic is given by $Q_{p} C_{c}$, while the rate of exhalation is given by $Q_{p} C_{x}$. Therefore we have the following equations:

$$
\begin{aligned}
\frac{d A_{i}}{d t} & =Q_{p} C_{c} \\
\frac{d A_{x}}{d t} & =Q_{p} C_{x}=Q_{p} \frac{C_{a}}{P_{b}} \\
\frac{d A_{L}}{d t} & =Q_{p}\left(C_{c}-C_{x}\right)+Q_{c}\left(C_{v}-C_{a}\right) .
\end{aligned}
$$




\section{PBPK Model for Inhaled TCE}

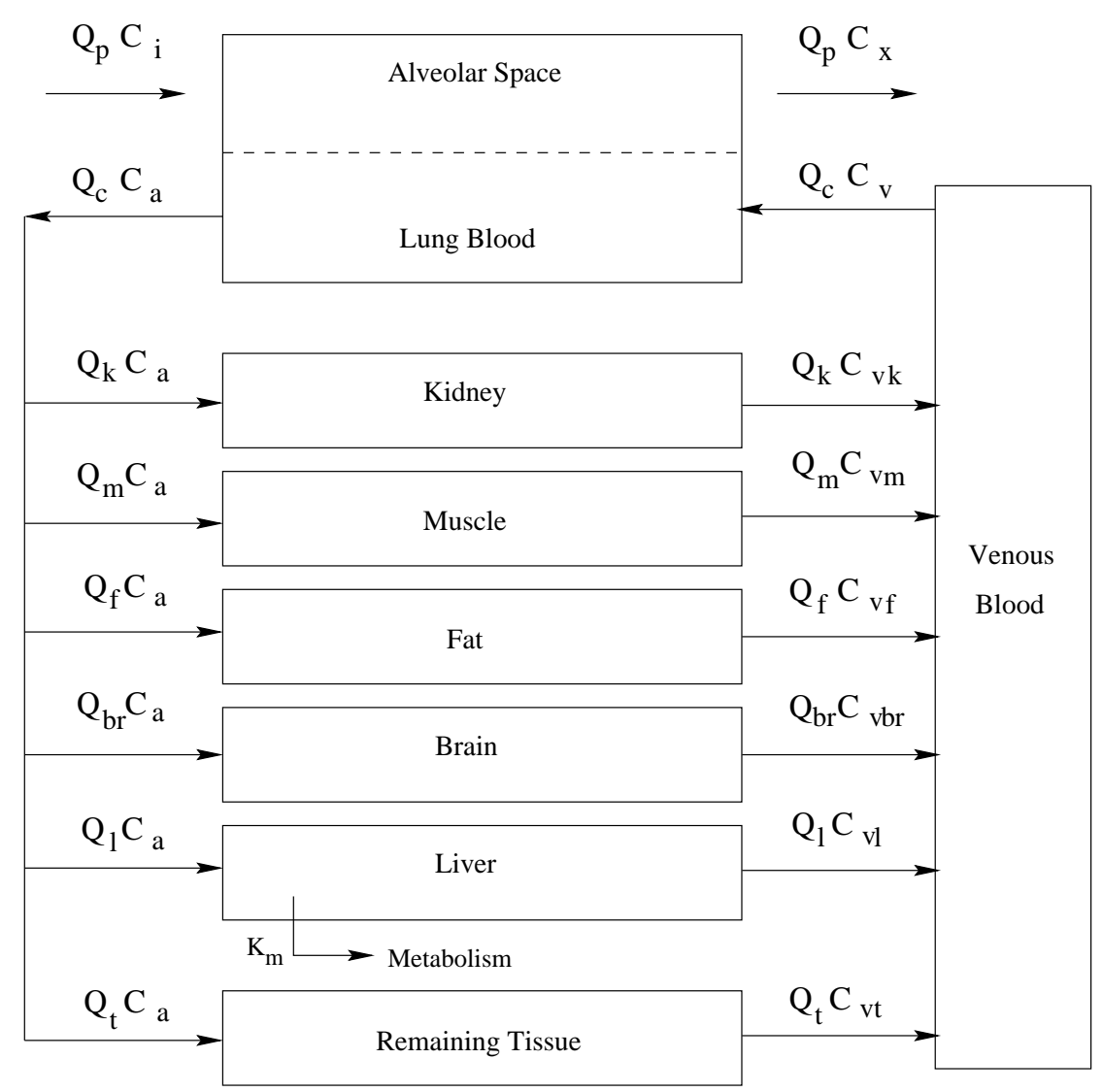

Figure 1: PBPK model for inhaled solute with flow-limited compartment for adipose tissue. 
Moreover, the assumptions of the model [38] imply that $\frac{d A_{L}}{d t}=0$, so by substituting $C_{x}=C_{a} / P_{b}$ we obtain

$$
C_{a}=\frac{Q_{p} C_{c}+Q_{c} C_{v}}{Q_{c}+\frac{Q_{p}}{P_{b}}}
$$

Combining the perfusion-limited compartments with the lung compartment, we obtain

$$
\begin{aligned}
V_{f} \frac{d C_{f}}{d t} & =Q_{f}\left(C_{a}-C_{v f}\right) \\
V_{v} \frac{d C_{v}}{d t} & =Q_{m} C_{v m}+Q_{t} C_{v t}+Q_{f} C_{v f}+Q_{b r} C_{v b r}+Q_{l} C_{v l}+Q_{k} C_{v k}-Q_{c} C_{v} \\
C_{a} & =\frac{Q_{c} C_{v}+Q_{p} C_{c}}{Q_{c}+\frac{Q_{p}}{P_{b}}} \\
V_{m} \frac{d C_{m}}{d t} & =Q_{m}\left(C_{a}-C_{v m}\right) \\
V_{t} \frac{d C_{t}}{d t} & =Q_{t}\left(C_{a}-C_{v t}\right) \\
V_{b r} \frac{d C_{b r}}{d t} & =Q_{b r}\left(C_{a}-C_{v b r}\right) \\
\frac{d A_{a m}}{d t} & =\frac{v_{m a x} C_{v l}}{k_{M}+C_{v l}} \\
V_{l} \frac{d C_{l}}{d t} & =Q_{l}\left(C_{a}-C_{v l}\right)-\frac{v_{m a x} C_{v l}}{k_{M}+C_{v l}} \\
V_{k} \frac{d C_{k}}{d t} & =Q_{k}\left(C_{a}-C_{v k}\right) \\
\frac{d A_{i}}{d t} & =Q_{p} C_{c} \\
\frac{d A_{x}}{d t} & =Q_{p} C_{x} .
\end{aligned}
$$

The subscripts denote the following specific tissues:

$$
\begin{aligned}
v & \Leftrightarrow \text { Venous blood } \\
k & \Leftrightarrow \text { Kidney } \\
m & \Leftrightarrow \text { Muscle } \\
f & \Leftrightarrow \text { Fat } \\
b r & \Leftrightarrow \text { Brain } \\
l & \Leftrightarrow \text { Liver } \\
t & \Leftrightarrow \text { Remaining non-fat tissue. }
\end{aligned}
$$

The amount of solute metabolized in the liver is denoted by $A_{a m}$, and has units in milligrams. Constants in the model include the Michaelis-Menten constant $k_{M}(\mathrm{mg} /$ liters $)$ and the metabolic constant $v_{\max }$ (mg/(hour)).

Volumes (in liters) of specific tissues are denoted by $V$, concentrations of solute (mg/liter) are denoted by $C$ and flow rates (liters/hour) are denoted by $Q$, each with subscripts corresponding to the specific tissue. The concentration of solute in the air is denoted by $C_{c}$, and is a specified quantity. The variables 


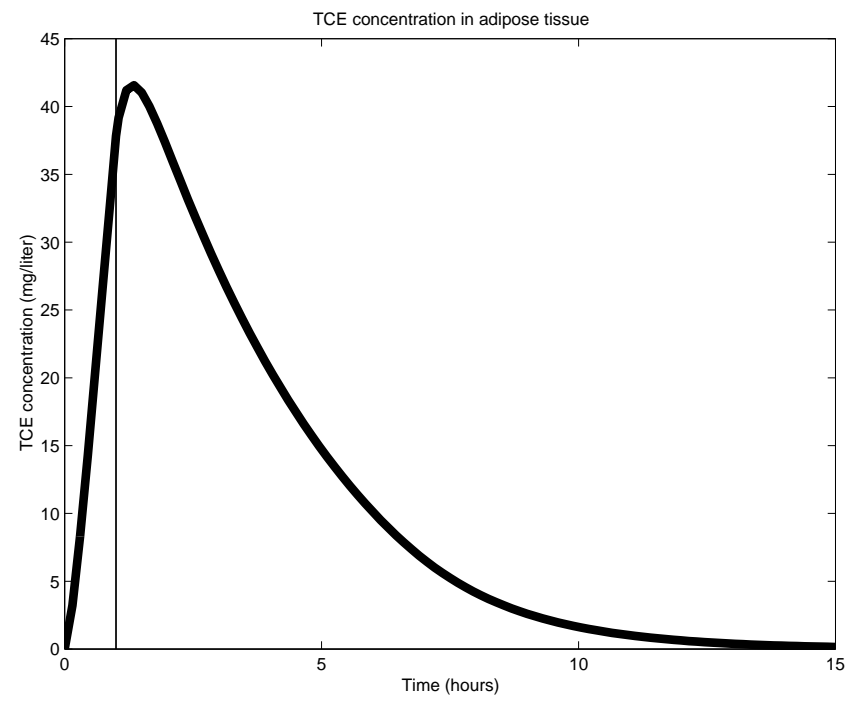

Figure 2: Model simulation: Concentration in time of unbound TCE inside the perfusion-limited fat compartment.

$C_{v k}, C_{v m}, C_{v f}, C_{v b r}, C_{v l}$ and $C_{v t}$ are the concentrations of solute leaving the respective organ and entering the venous blood system. In this case, all compartments except for the lung are perfusion-limited, so the concentration of solute leaving each of these compartments is equal to the concentration of free solute in that compartment itself. In the kidney, for example, this implies

$$
C_{v k}=\frac{C_{k}}{P_{k}}
$$

where $C_{k}$ is the total concentration of solute inside the kidney compartment and $P_{k}$ is the tissue/blood partition coefficient for the kidney.

We note that our model includes a venous blood compartment governed by the differential equation (2). In this type of PBPK model, the algebraic equation

$$
C_{v}=\frac{1}{Q_{c}}\left(Q_{m} C_{v m}+Q_{t} C_{v t}+Q_{f} C_{v f}+Q_{b r} C_{v b r}+Q_{l} C_{v l}+Q_{k} C_{v k}\right)
$$

is often used and is generally sufficient to capture the behavior of the solute in the venous blood. This equation is based on a steady-state assumption for the large venous blood compartment. Here we include the more complicated model (2) because it will be more compatible with our modified model which uses a dispersion compartment for the adipose tissue.

Simulations were carried out using the model (1) - (11) to predict TCE concentrations in Long-Evans rats. The Matlab code for this model and the model parameters were provided by Evans et al. [22]. Figure 2 depicts an example simulation for the following input function: $2000 \mathrm{ppm}$ TCE in the surrounding air for one hour, followed by $0 \mathrm{ppm}$ TCE in the surrounding air for 14 hours. The concentration of unbound TCE inside the perfusion-limited fat compartment is given in Figure 2. Note the rapid, exponential decay of concentrations following $t=1$ hour. This simulation illustrates that this type of compartmental model may not be able to capture the slow accumulation and release of TCE in the adipose tissue. 


\subsection{A PBPK model for TCE with a diffusion-limited adipose tissue compart- ment}

A second type of compartment used in PBPK modeling is the diffusion-limited, or membrane-limited compartment. Unlike the perfusion-limited model, the blood flow rate to the tissue is rapid compared to the transport of the compound across the cell membranes [38]. This compartment is divided into two subcompartments: the intracellular space and the extracellular space, which includes the vascular blood and the interstitial space. The concentration of compound within each of these subcompartments is in equilibrium, and the equations governing transport of the compound through the tissue are

$$
\begin{aligned}
V_{E} \frac{d C_{E}}{d t} & =Q\left(C_{\text {in }}-C_{\text {out }}\right)+\mu\left(C_{I}-C_{E}\right) \\
V_{I} \frac{d C_{I}}{d t} & =\mu\left(C_{E}-C_{I}\right)
\end{aligned}
$$

where $V_{E}$ and $V_{I}$ are the volumes of the extracellular and intracellular compartments, respectively, and $C_{E}$ and $C_{I}$ are the concentrations of compound in the extracellular and intracellular compartments, respectively. Here $\mu$ is the cell membrane permeability for the compound (in liters/hour), and $Q, C_{\text {in }}$, and $C_{\text {out }}$ are defined as above.

Now we modify the previous PBPK model for TCE to include a diffusion-limited fat compartment (see Figure 3). The remainder of the model is identical, but for fat we assume that the flow of blood through adipose tissue is much faster than the diffusion of solute across the cell membranes. Thus, we replace (1) by

$$
\begin{aligned}
V_{f e} \frac{d C_{f e}}{d t} & =Q_{f}\left(C_{a}-C_{v f}\right)+\mu\left(C_{f i}-C_{f e}\right) \\
V_{f i} \frac{d C_{f i}}{d t} & =\mu\left(C_{f e}-C_{f i}\right)
\end{aligned}
$$

where $C_{f e}$ and $C_{f i}$ are the concentrations of solute in the extracellular and intracellular spaces of the fat tissue, respectively, $V_{f e}$ and $V_{f i}$ are the volumes of these extracellular and intracellular spaces, and $\mu$ is the cell membrane permeability coefficient for the solute. Our new system of equations is therefore given by (12), (13) and (2) - (11).

Model simulations were carried out for this model as before. The concentrations of TCE in the adipose subcompartments are shown in Figure 4 for varying values of $\mu$. For $\mu=1$, the concentration of unbound TCE in the extracellular subcompartment as seen in Figure 4 (Right) is nearly identical to the concentration of unbound TCE in the perfusion-limited adipose compartment (Figure 2).

As $\mu$ decreases, the peak concentration of unbound TCE in the intracellular subcompartment decreases and the decay following exposure is less rapid. The concentration profile in the intracellular space for $\mu=0.1$ shows a steady increase in TCE concentrations before $t=1$ hour, followed by a slow decrease. The dynamics for this value of $\mu$ appear to match more closely with the expected behavior of TCE in the adipose tissue than the dynamics for the perfusion-limited compartmental model.

One disadvantage with the diffusion-limited model, however, is that there is only one extra degree of freedom and there is little flexibility in obtaining both the correct concentration profile and the correct peak value in the intracellular compartment. Moreover, since TCE has a low molecular weight (131.3889) and is highly lipophilic, it diffuses easily across cell membranes. This diffusion rate will be much greater than the blood flow rate to the adipose tissue, which suggests that the diffusion-limited model may not be physically appropriate. 


\section{PBPK Model for Inhaled TCE}

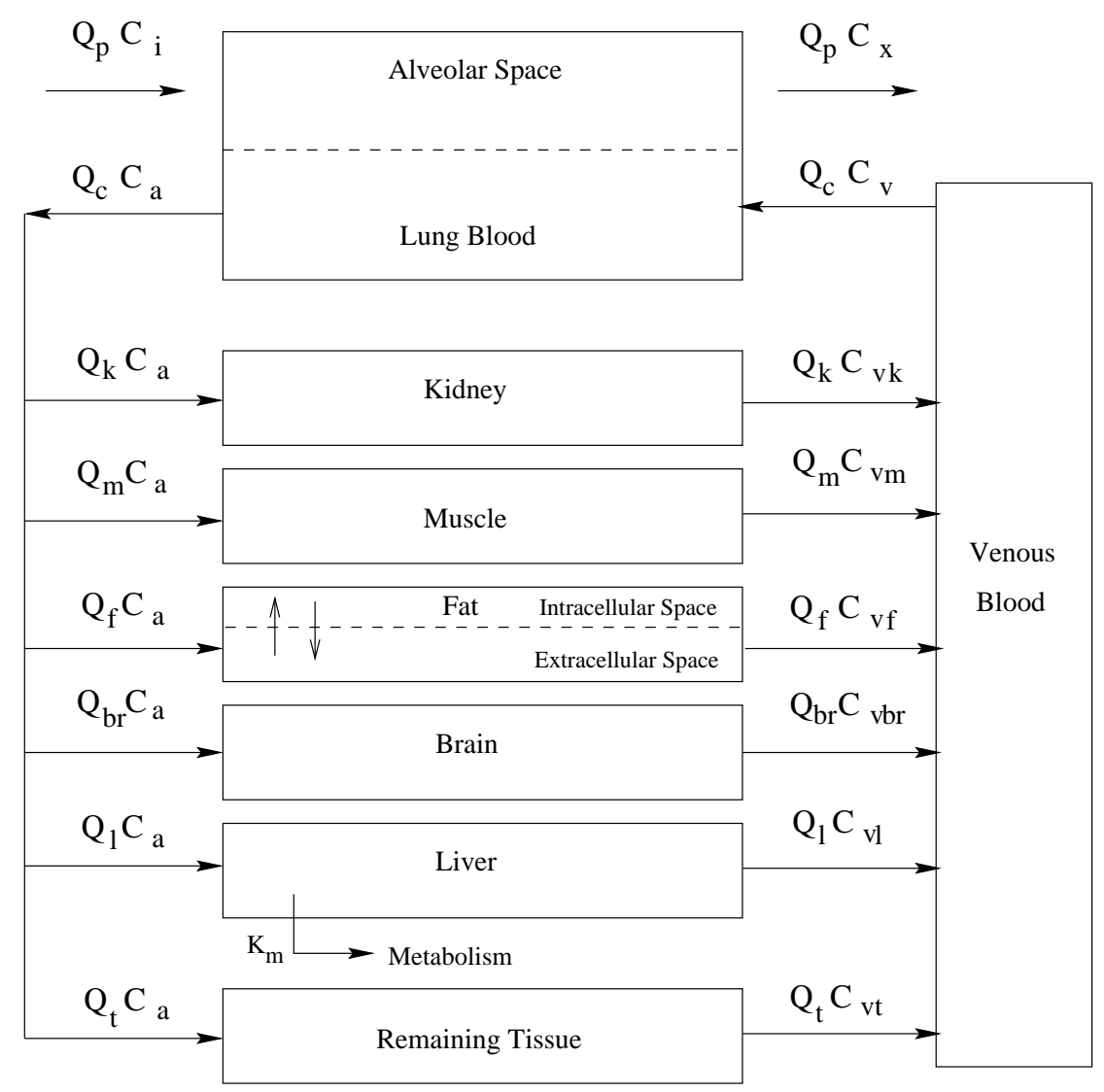

Figure 3: PBPK model for inhaled solute with membrane-limited compartment for adipose tissue. 

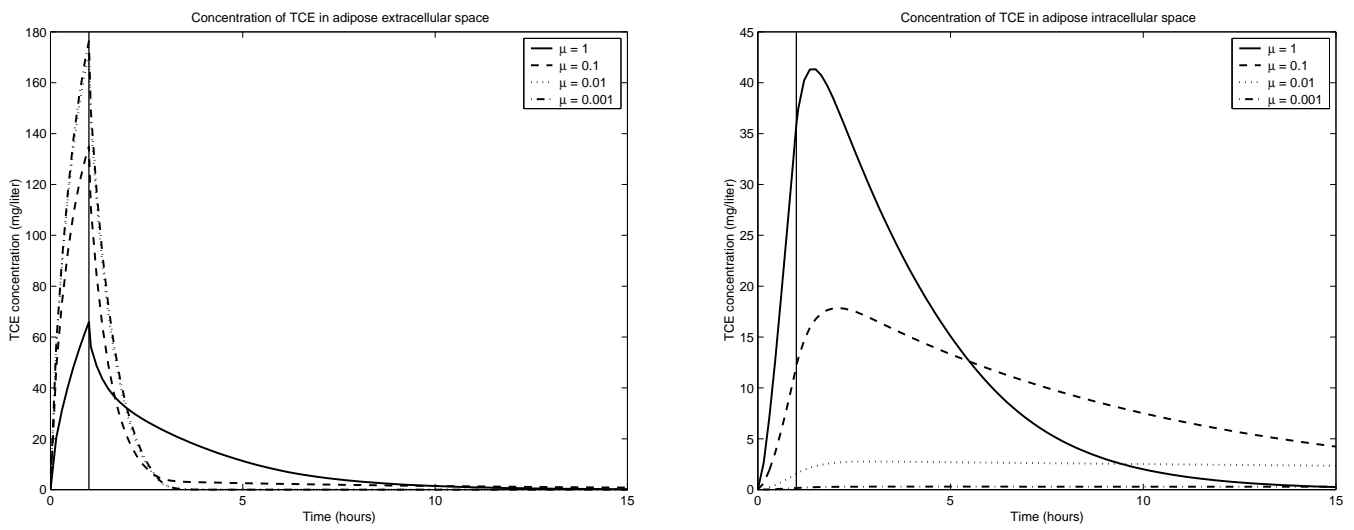

Figure 4: Model simulation: Concentrations of TCE in time in the diffusion-limited fat compartment for various values of the permeability coefficient $\mu$. Left: Total concentration of TCE in the extracellular subcompartment. Right: Concentration of unbound TCE in the intracellular subcompartment.

\section{The physiology of adipose tissue and the dispersion model}

Neither the perfusion-limited nor the diffusion-limited compartmental models are designed to account for spatial heterogeneities within the intracellular space of a given tissue. Adipose tissue is known to be widely heterogeneous in both its composition and functional properties. Its general structure includes a rich, irregular vascular bed, with capillaries in contact with each adipocyte, or fat cell [47]. The adipocytes, relatively large spherical cells containing lipid droplets that make up the bulk of each cell, are organized into a structure of lobules supported by connective tissue [47]. See Figure 5 for an illustration of a representative adipocyte and capillary unit, which is suspended in the surrounding interstitial fluid. An example scanning electron micrograph of adipose tissue that is on the same scale as the illustration can be found in [49], p. 92.

The major metabolic functions of adipose tissue include: the synthesis and storage of lipid in the form of triglyceride [17], the breakdown of triglyceride and the mobilization of lipid in the form of free fatty acids [17], and the uptake of fatty acids, glucose and amino acids [47].

Unlike many organs, the adipose "organ" is located in multiple distinct depots throughout the body. Several studies have shown that there are pronounced differences in tissue composition, blood flow and metabolic activities across these depots [17], [19], [20], [37], [51], which suggest that a single perfusion-limited or diffusion-limited compartment may be insufficient to capture the behavior of adipose tissue as a whole. A possible modification to these models is the inclusion of many separate perfusion-limited or diffusion-limited compartments, each representing a distinct adipose depot and each with its own physiological parameters.

In addition to inter-depot differences in blood flow, metabolism and tissue composition, there are heterogeneities within each adipose depot itself. The sizes of individual fat cells greatly vary, as does the amount of lipid in each adipocyte, creating an uneven distribution of lipids across the tissue [32]. Since TCE and other compounds of interest are highly lipophilic, an uneven distribution of these compounds may result, leading to a spatially dependent concentration function inside the adipose tissue.

It has been shown that the metabolic activities of adipose tissue are directly linked to its blood flow properties [17]. As the metabolic process varies in different regions of tissue and in time, the perfusion of blood to those regions changes to respond to the needs of the tissue at that moment. Moreover, the blood flow to adipose tissue is directly affected by the local concentrations of substrates and hormones used in the process of metabolizing lipids [19]. Blood flow in adipose tissue is also controlled by the central 


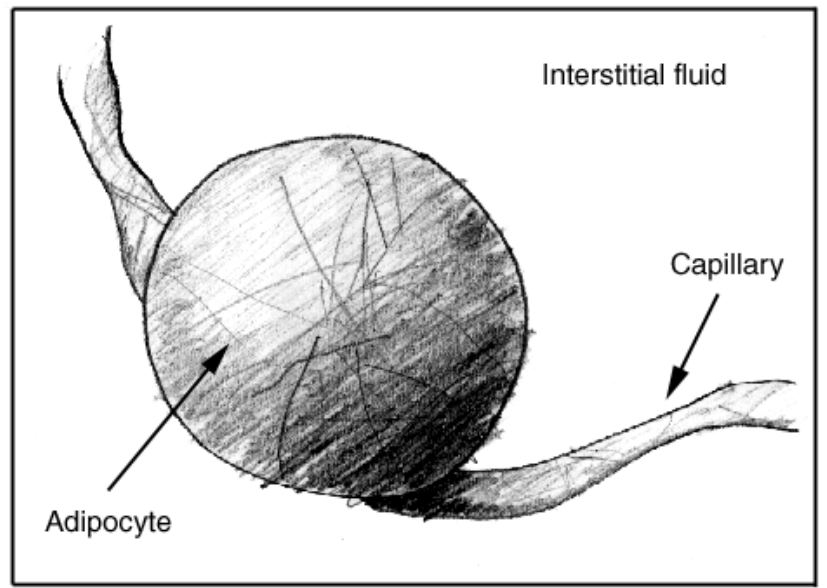

Figure 5: Representation of an adipocyte and capillary, surrounded by interstitial fluid.

nervous system, which can alter vascular permeability, capillary density, diffusion, and the rate of transport of lipophilic compounds [42]. Together, these physiological characteristics of adipose tissue suggest that a spatially varying model may be more appropriate for describing the disposition of lipophilic xenobiotics through adipose tissue.

\subsection{Modeling philosophy}

An important feature of adipose tissue is that each adipocyte and connecting capillary is an individual functional "unit" within the tissue. There are millions of fat cells within adipose tissue, and it would be nearly impossible to capture the disposition of TCE specifically through each and every adipocyte. Rather, there are several "aggregate" modeling approaches that may be used to approximate the transport through the tissue without actually modeling each cell.

One such approach utilizes mathematical homogenization theory, as used in [45] and [46] to estimate the magnetic permeability of magnetorheological fluids. The homogenization technique involves a domain with a periodic microstructure, such as a collection of individual fat cells with capillaries immersed in interstitial fluid, resulting in an "effective" equation that approximates the overall behavior inside the domain. In the case of adipose tissue, such a model would approximate the effective concentration of TCE inside the tissue.

Another modeling approach designed to handle this type of heterogeneity makes use of probability theory. As there are widely varying ranges for parameters such as fat cell size, blood flow rates and cell permeability coefficients inside the fat tissue, we could think of each of these quantities as a random variable. Each of these random variables would have an unknown probability distribution that could be estimated with inverse problem techniques. See [3] and [4] for detailed descriptions of this modeling philosophy.

A third type of model sometimes used in physiological modeling is the axial dispersion model, which is designed to account for the heterogeneity of tissue. It assumes that the amount of time it takes for a compound to pass through a given tissue is not constant for each particle of compound. The dispersion model is based on the premise that the variations in these "residence times" are a result of radial variations in flow velocity, variations in path length, convective mixing in the direction of blood flow, and molecular diffusion.

One important feature of this model is that it is an aggregate type of model, using one representative 
"cell" to capture the behavior of a large collection of similar "cells" that have varying properties. The variability among these cells is accounted for in the model with an axial dispersion term, and the axial dispersion coefficient is a representative measure of the variability within the overall system. See [35] for a detailed development of the axial dispersion model.

Roberts and Rowland [41] adapted this type of dispersion model for the flow of compounds through the liver. Based on this model, Banks, Musante and Tran [6] developed a dispersion model for the transport and elimination of 2,3,7,8-tetrachlorodibenzo-p-dioxin in the liver. The complex, heterogeneous physiology of the liver and the widely varying circulation of blood through the liver's sinusoids both suggest this type of model, and simulations carried out in [5] illustrate the effectiveness of this model for describing transport through the liver.

\section{A dispersion model for TCE in adipose tissue}

Here we develop an axial dispersion-type model for the adipose tissue compartment, which will connect with the remaining PBPK compartmental model. This model is based on the dispersion model found in [35] and will be adapted for a representative geometry for adipose tissue.

\subsection{Geometry}

As we have discussed, adipose tissue is composed primarily of adipocytes, which are relatively large sphericalshaped cells. The adipocytes are supported with a network of connective tissue, and each fat cell is in contact with one or more capillaries. The interstitial space makes up about $10 \%$ of the total tissue weight of adipose tissue.

Based on these histological characteristics, we choose a representative geometry for the fat compartment that includes three subcompartments: the capillary subcompartment, the adipocyte subcompartment and the interstitial fluid compartment. We choose to represent the adipocyte subcompartment by a single sphere centered at the origin (see Figure 6). The adipocyte is in contact with a capillary, represented by a cylindrical tube with a circular cross section. The space surrounding the capillary and the adipocyte is occupied by the interstitial fluid, including the space between the capillary and adipocyte.

The adipocyte region is denoted by (A), and consists of the sphere centered at the origin with radius $r_{1}$. The capillary or blood region (B) is a cylindrical tube with a circular cross-section that wraps around the capillary. This cylindrical tube (again see Figure 6) has a central axis $r=r_{2}+\alpha, \theta=\theta_{0}, \varepsilon_{1}<\phi<\pi-\varepsilon_{2}$ in spherical coordinates, where $r_{2}>r_{1}>0$ and $\varepsilon_{1}, \varepsilon_{2}>0$. The cross section of the capillary has radius $\alpha$ and area $A_{B}$. The domain of this cylindrical tube is denoted by $\Omega_{B}$. The capillary connects with the arterial blood at $\phi=\varepsilon_{1}$, and similarly connects with the venous blood at $\phi=\pi-\varepsilon_{2}$. The interstitial fluid region (I) is the space surrounding the adipocyte and the capillary, including the space between the capillary and adipocyte $\left(r_{1}<r<r_{2}\right)$.

\subsection{Model assumptions}

Blood flows from the arterial compartment into the capillary, carrying TCE to the adipose tissue. TCE then diffuses back and forth across the capillary endothelium into and out of the interstitial fluid, and TCE in the interstitial fluid may also diffuse back and forth across the plasma membrane of the adipocyte into and out of the fat cell. At the exit to the capillary the blood and TCE flow into the venous blood compartment. See Figure 6 for a schematic of the assumed geometry. 

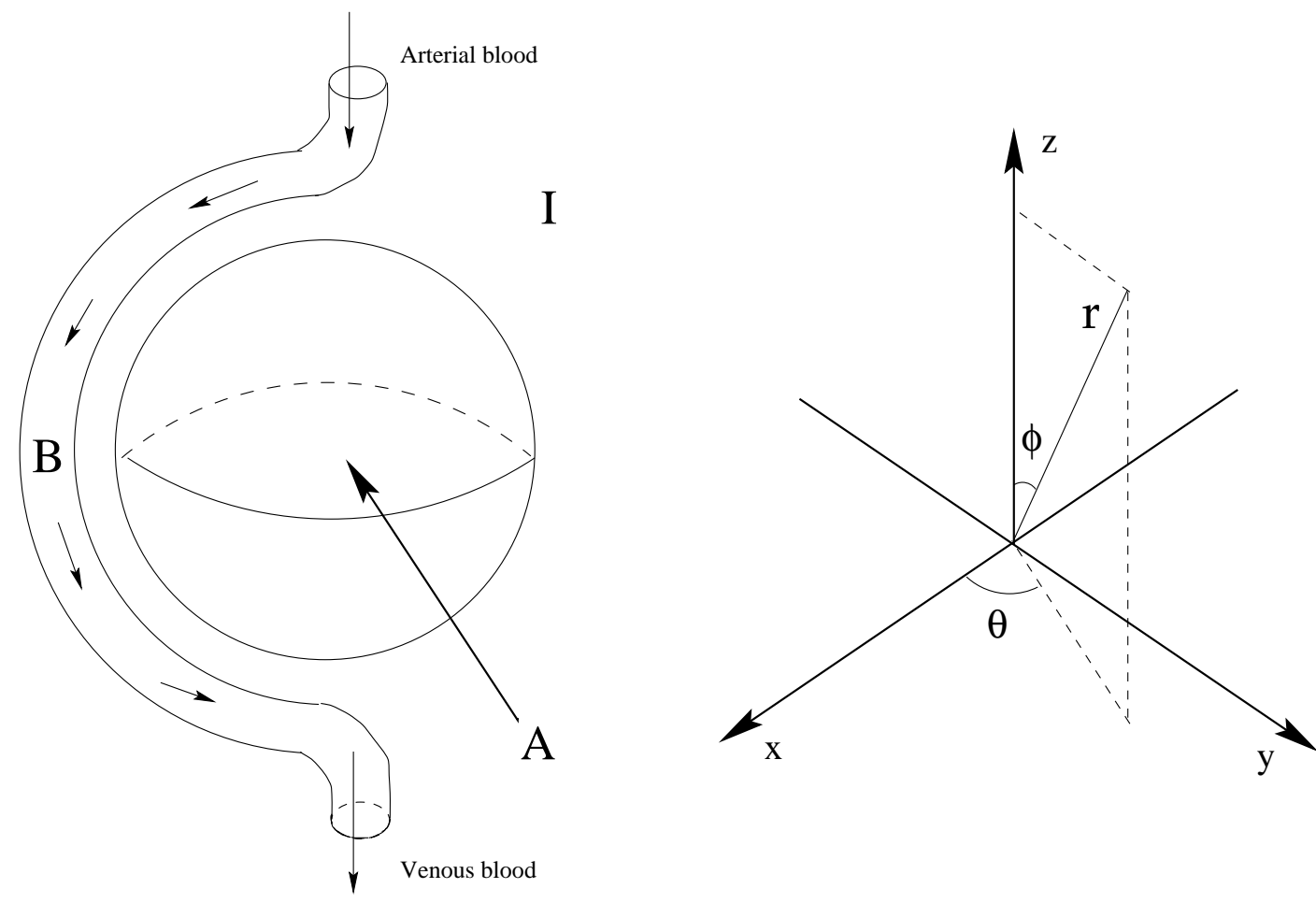

Figure 6: Adipose tissue represented geometrically: the adipocyte region (A) is a sphere, surrounded by the interstitial space (I). The capillary or blood region (B) is a cylindrical tube that wraps around the capillary. Coordinates are in spherical coordinates $(r, \theta, \phi)$. 
Some studies have suggested that there may be an "interfacial continuum" between capillary endothelial cells and adipocytes [9], [10], [11], [44]. That is, there is evidence of membrane continuity between the membranes of capillary endothelial cells and the plasma membranes of adipocytes. Since TCE is highly lipophilic and the cell membranes are composed of lipid bilayers [31], TCE may diffuse directly across the capillary endothelium and the adipocyte plasma membrane at these areas of membrane continuity. This would result in direct transport between the capillary and the adipocyte.

Therefore there are two feasible routes of transport between the capillary and the adipocyte: indirect transport via diffusion through the interstitial fluid and direct transport across an "interfacial continuum." It would be difficult to determine in vivo the exact proportions of direct and indirect transport of TCE occurring between an adipocyte and a capillary. Our model will include both of these types of transport, and the proportions of each type will be parameters that may be estimated using inverse problem techniques.

We note that here we do not attempt to include in our representative geometry the areas of continuity between the capillary endothelium and the adipocyte plasma membrane. Rather than modeling each of these regions individually, we will include a general term in our model that will account for any direct transport that occurs.

We assume that the concentrations of TCE in the interstitial fluid are in equilibrium at points far away from the adipocyte and the capillary. Specifically, we assume that the net radial flux of TCE across the spherical shell $r=r_{3}$ is equal to zero for some $r_{3}>r_{2}+2 \alpha$, where $r_{2}+2 \alpha$ is the outer radius of the capillary. We define this region of the interstitial space as

$$
\Omega_{I}=\left\{(r, \theta, \phi): r_{1} \leq r \leq r_{3}, 0 \leq \theta \leq 2 \pi, 0<\phi<\pi\right\} .
$$

Similarly, since the adipocyte is large relative to the size of the capillary and nearby interstitial fluid, we assume that the net radial flux of TCE inside the adipocyte across the spherical shell $r=r_{0}$ is equal to zero for some $0<r_{0}<r_{1}$. We thus define the adipocyte domain by

$$
\Omega_{A}=\left\{(r, \theta, \phi): r_{0} \leq r \leq r_{1}, 0 \leq \theta \leq 2 \pi, 0<\phi<\pi\right\} .
$$

Here we assume that the difference between the inner radius $r_{0}$ and the outer radius $r_{3}$ are small compared to the radius $r_{1}$ of the adipocyte.

The remaining assumptions for our dispersion model are based on those used by Roberts and Rowland for the liver, adapted here for the physiology of adipose tissue.

1. Conditions in the capillary do not vary in a cross section normal to flow. This implies that the concentration $C_{B}$ of TCE in the capillary depends only on $\phi$. The basis for this assumption is that the flow in the $r$ and $\theta$ directions is negligible compared to the flow of the blood in the $\phi$ direction.

2. Axial diffusion of solute is slow compared to the convection of solute in the capillary, so it may be ignored. It can be shown that without loss of generality, a separate axial diffusion term can be omitted in any axial dispersion model. If we assume axial diffusion, then we obtain the following diffusive mass flux in the $\phi$ direction:

$$
J_{\phi}=-D \frac{\partial C}{\partial \phi}
$$

where $C$ is the concentration of solute and $D$ is the axial diffusivity coefficient. When considering axial dispersion, the dispersive mass flux in the $\phi$ direction is given by

$$
J_{\phi}=-\mathcal{D} \frac{\partial C}{\partial \phi}
$$

where $\mathcal{D}$ is the axial dispersivity coefficient. Since neither of these coefficients are known in general, they must be estimated using inverse problem techniques. Indeed, the dispersivity coefficient is not physical, 
since it is an effective coefficient which accounts for variations in residence times, path length, local geometry, etc. Therefore we can interpret the coefficient of this flux term as either the axial diffusivity, axial dispersivity, or as a net axial transport coefficient involving a combination of the two.

3. The transport of TCE between the capillary and the interstitial space is assumed to obey Fick's law of diffusion, as are the transport between the interstitial space and the adipocyte and the transport between the capillary and the adipocyte.

4. No metabolism or elimination of solute occurs in adipose tissue. This assumption is based on the fact that TCE is metabolized by the enzyme Cytochrome P450 2E1, which is located primarily in the liver and is not known to be present at significant levels inside adipose tissue. Moreover, TCE is eliminated only via exhalation from the lungs and via the excretion of its metabolites in the urine.

5. Only unbound solute can cross cell membranes. This is a standard assumption used in physiological modeling.

6. Concentrations of TCE in the interstitial fluid immediately surrounding the capillary and adipocyte $\left(r_{1}<r<r_{3}\right)$ are uniform with respect to $r$, depending only on $\theta$ and $\phi$. This assumption is based on the small radial distance between $r_{1}$ and $r_{3}$.

7. Concentrations of TCE in the interstitial fluid near the capillary vary only slightly with respect to $\theta$, and the transport between the capillary and the interstitial space is approximated by the transport occurring at $r=r_{2}, \theta=\theta_{0}, \varepsilon_{1}<\phi<\pi-\varepsilon_{2}$.

8. Concentrations of TCE inside the adipocyte near the outer radius $r_{1}$ are uniform with respect to $r$, depending only on $\theta$ and $\phi$.

9. The ratio $f_{B}$ of the unbound solute to the total solute concentration within the blood is a constant. This is also a standard physiological assumption, based on the fact that the total and unbound concentrations of solute in the blood are in equilibrium with each other, and a linear relationship between the free and unbound concentrations is assumed. Let $C_{B}$ and $C_{u_{B}}$ denote the blood concentrations of total and unbound solute, respectively. Then

$$
C_{u_{B}}=f_{B} C_{B}
$$

for some $f_{B}$, and the fact that these concentrations are in equilibrium implies that $f_{B}$ must be a constant.

10. The ratio $f_{I}$ of the unbound solute to the total solute concentration within the interstitial space is a constant. This assumption is the interstitial space analog of Assumption 9.

11. The ratio $f_{A}$ of the unbound solute to the total solute concentration within the adipocytes is a constant. This assumption is the adipocyte analog of Assumption 9.

\subsection{Derivation of equations: Capillary region}

Consider a "spherical cuboid" volume element spanning from $r$ to $r+\Delta r, \theta$ to $\theta+\Delta \theta$ and $\phi$ to $\phi+\Delta \phi$ (see Figure 7). The volume of this element is $\Delta V=r^{2} \sin \phi \Delta r \Delta \phi \Delta \theta$, and we will denote its surface by $\Delta S$.

In the capillary, the concentration of TCE is uniform with respect to both $r$ and $\theta$, and we have axial dispersion in the $\phi$ direction. Moreover, we assume that $\alpha$ is small relative to $r_{2}$ so that we may approximate $r \approx r_{2}$ for $r \in \Omega_{B}$. This implies that the flux vector $J=\left(J_{r}, J_{\theta}, J_{\phi}\right)$ is given by

$$
J=\left(0,0,-\frac{\mathcal{D}_{B}}{r_{2}} \frac{\partial C_{B}}{\partial \phi}+v C_{B}\right),
$$




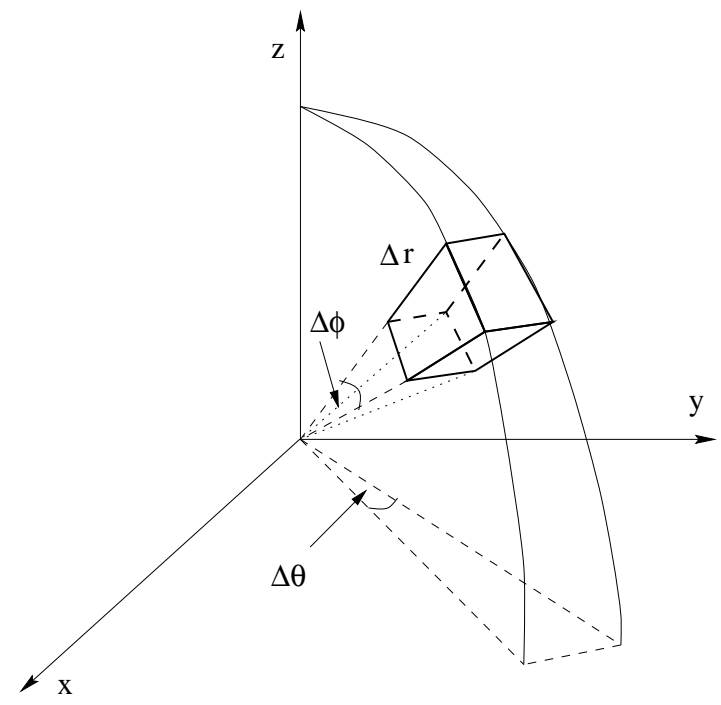

Figure 7: "Spherical cuboid" volume element.

where $\mathcal{D}_{B}$ is the axial dispersion coefficient with units $\mathrm{m}^{2} / \mathrm{hr}$. The velocity $v$ is given by

$$
v=\frac{Q_{f}}{1000 A_{B}}=\mathcal{F}\left(Q_{f} \frac{\text { liters }}{\text { hour }}\right)\left(\frac{1}{A_{B} \text { meters }^{2}}\right)\left(\frac{1 \text { meter }^{3}}{1000 \text { liters }}\right)
$$

with units $\mathrm{m} / \mathrm{hr}$, where $Q_{f}$ is the blood flow rate to the fat tissue in liters $/ \mathrm{hr}$ and $A_{B}$ is the cross-sectional area of the capillary in meters ${ }^{2}$.

The dimensionless coefficient $\mathcal{F}$ is included to account for the inversion of the Fahraeus-Lindquist effect, which is a phenomenon that involves the flow of blood as a function of the vessel diameter. To understand the phenomenon involved, consider the flow of blood from a larger vessel to a smaller vessel, with the smaller vessel ranging between 15 to 500 microns in diameter. If we assume that blood with constant hematocrit (the fraction of blood volume made up of the red blood cells) flows from the larger vessel to the smaller vessel, the hematocrit in the smaller vessel decreases as the diameter of the vessel decreases [27]. This is known as the Fahraeus effect, and is related directly to the Fahraeus-Lindquist effect, which involves a concurrent decrease in the apparent viscosity of blood in the smaller vessel as the diameter and the hematocrit decrease [27].

The apparent viscosity $\mu_{a p p}$ can be expressed as a function of the vessel radius $R$ and length $L$, the drop in pressure $\Delta P$ across the length of the vessel, and the volumetric flow rate $Q$ as in [26] and [27]:

$$
\mu_{\text {app }}=\frac{\pi R^{4} \Delta P}{8 L Q} .
$$

A different phenomenon occurs in vessels less than about 4-6 microns in diameter. These vessels are significantly smaller than the normal 8 micron diameter of red blood cells, making it difficult for the red blood cells to enter vessels of this size [27]. This results in an increase of the flow resistance and hence an increase in the apparent viscosity of the blood as the diameter of the vessel decreases, which is the inversion of the Fahraeus-Lindquist effect [27]. This phenomenon leads to a concurrent decrease in the flow rate of the blood inside the smaller tube compared to the flow rate in the larger feed vessel.

The capillaries in adipose tissue typically have lumen diameters measuring between 2 and 4 microns [19], which would place them in the range of vessels covered by the inversion of the Fahraeus-Lindquist effect. This 
implies a significant difference between the flow velocities of blood inside the arterial blood system versus the velocities inside the capillaries of the adipose tissue. Hence we include the "reverse Fahraeus-Lindquist parameter" $\mathcal{F}<1$ to account for this decrease in blood flow rates as the blood and TCE flow from the arterial vessels to the adipose capillaries.

Conservation of mass implies that

$$
\begin{aligned}
\left(\begin{array}{c}
\text { Net rate of change } \\
\text { of TCE in blood element }
\end{array}\right) & =\left(\begin{array}{c}
\text { Net flow rate of TCE } \\
\text { within blood element }
\end{array}\right)+\left(\begin{array}{c}
\text { Net flow rate of TCE } \\
\text { between blood element } \\
\text { and interstitial space }
\end{array}\right) \\
& +\left(\begin{array}{c}
\text { Net flow rate of TCE } \\
\text { between blood element } \\
\text { and adipocyte region }
\end{array}\right) .
\end{aligned}
$$

First we consider only transport from within the capillary (i.e., no exchange with the interstitial space or adipocyte region). Then

$$
\left(\begin{array}{c}
\text { Net flow rate of TCE } \\
\text { within blood element }
\end{array}\right)=\iint_{\Delta S}-J \cdot \hat{n} d S
$$

where $\hat{n}$ is the outer unit normal to the surface $\Delta S$. Dividing by the volume $\Delta V$ of the element, we obtain

$$
\begin{aligned}
\left(\begin{array}{c}
\text { Net flow rate of TCE } \\
\text { within blood element } \\
\text { per unit volume }
\end{array}\right) & =\frac{1}{\Delta V} \iint_{\Delta S}-J \cdot \hat{n} d S \\
& =\frac{1}{\Delta V} \iiint_{\Delta V} \operatorname{Div}(-J) d V
\end{aligned}
$$

by the Divergence Theorem. Taking the limit as $\Delta V \rightarrow 0$ at $r=r_{2}$, we have

$$
\begin{aligned}
\left(\begin{array}{c}
\text { Net flow rate of TCE } \\
\text { in the blood element } \\
\text { per unit volume } \\
\text { at the point }\left(r_{2}, \theta, \phi\right)
\end{array}\right) & =\operatorname{Div}(-J) \\
& =-\frac{1}{r_{2}^{2}} \frac{\partial}{\partial r}\left(r_{2}^{2} J_{r}\right)-\frac{1}{r_{2} \sin \phi} \frac{\partial J_{\theta}}{\partial \theta}-\frac{1}{r_{2} \sin \phi} \frac{\partial}{\partial \phi}\left(\sin \phi J_{\phi}\right) \\
& =-\frac{1}{r_{2} \sin \phi} \frac{\partial}{\partial \phi}\left(\sin \phi J_{\phi}\right) \\
& =-\frac{1}{r_{2} \sin \phi} \frac{\partial}{\partial \phi}\left[\sin \phi\left(-\frac{\mathcal{D}_{B}}{r_{2}} \frac{\partial C_{B}}{\partial \phi}+v C_{B}\right)\right]
\end{aligned}
$$

Multiply both sides by the volume of the capillary $V_{B}$ to obtain

$$
\left(\begin{array}{c}
\text { Net flow rate of TCE } \\
\text { within capillary }
\end{array}\right)=\frac{V_{B}}{r_{2} \sin \phi} \frac{\partial}{\partial \phi}\left[\sin \phi\left(\frac{\mathcal{D}_{B}}{r_{2}} \frac{\partial C_{B}}{\partial \phi}-v C_{B}\right)\right] .
$$

Now consider the exchange of TCE between the capillary and interstitial space. By Assumption 3, this transport is governed by Fick's law of diffusion, so that

$$
\left(\begin{array}{c}
\text { Net flow rate of TCE } \\
\text { between blood element } \\
\text { and interstitial space }
\end{array}\right)=\lambda_{I} \mu_{B I}\left(f_{I} C_{I}\left(\theta_{0}\right)-f_{B} C_{B}\right)
$$


where $\mu_{B I}$ is the permeability coefficient for transport between the capillary and interstitial regions, with units liters/hour. The dimensionless coefficient $\lambda_{I}$ represents the fraction of transport that occurs between the capillary and interstitial fluid (versus the direct transport between the capillary and the adipocyte).

Similarly, we model the transport between the capillary and the adipocyte by

$$
\left(\begin{array}{c}
\text { Net flow rate of TCE } \\
\text { between blood element } \\
\text { and adipocyte }
\end{array}\right)=\lambda_{A} \mu_{B A}\left(f_{A} C_{A}\left(\theta_{0}\right)-f_{B} C_{B}\right),
$$

where $\mu_{B A}$ is the permeability coefficient for transport between the capillary and adipocyte regions (in liters/hour), and $\lambda_{A}$ represents the fraction of transport that occurs between the capillary and the adipocyte. Note that we have $\lambda_{I}+\lambda_{A}=1$.

Since the total rate of change of TCE at a point in the blood element is given by $V_{B} \frac{\partial C_{B}}{\partial t}$, we have $V_{B} \frac{\partial C_{B}}{\partial t}=\frac{V_{B}}{r_{2} \sin \phi} \frac{\partial}{\partial \phi}\left[\sin \phi\left(\frac{\mathcal{D}_{B}}{r_{2}} \frac{\partial C_{B}}{\partial \phi}-v C_{B}\right)\right]+\lambda_{I} \mu_{B I}\left(f_{I} C_{I}\left(\theta_{0}\right)-f_{B} C_{B}\right)+\lambda_{A} \mu_{B A}\left(f_{A} C_{A}\left(\theta_{0}\right)-f_{B} C_{B}\right)$ for $t \geq 0$ and $(r, \theta, \phi) \in \tilde{\Omega}_{B}$, where

$$
\tilde{\Omega}_{B}=\left\{(r, \theta, \phi): r=r_{2}, \theta=\theta_{0}, \varepsilon_{1}<\phi<\pi-\varepsilon_{2}\right\} \subset \Omega_{B} .
$$

Note that $C_{B}=C_{B}(t, \phi)$ due to uniformity in $r$ and $\theta$.

\subsubsection{Boundary conditions}

To obtain boundary conditions for the capillary region, we use flux balance principles. The mass flux in the capillary across the boundary $\phi=\varepsilon_{1}$ is given by $\left.J_{\phi}\right|_{\phi=\varepsilon_{1}}$, and the mass flux in the arterial blood across $\phi=\varepsilon_{1}$ is $\left(Q_{c} /\left(1000 A_{B}\right)\right) C_{a}$. Therefore we have

$$
-\frac{\mathcal{D}_{B}}{r_{2}} \frac{\partial C_{B}}{\partial \phi}(t, \phi)+\left.v C_{B}(t, \phi)\right|_{\phi=\varepsilon_{1}}=\frac{Q_{c}}{1000 A_{B}} C_{a}(t) .
$$

Similarly, using flux balance at the boundary $\phi=\pi-\varepsilon_{2}$ between the capillary and the venous blood, we obtain

$$
-\frac{\mathcal{D}_{B}}{r_{2}} \frac{\partial C_{B}}{\partial \phi}(t, \phi)+\left.v C_{B}(t, \phi)\right|_{\phi=\pi-\varepsilon_{2}}=\frac{Q_{c}}{1000 A_{B}} C_{v}(t)
$$

\subsection{Interstitial space}

In the interstitial space, TCE enters and exits via diffusion from the capillary region and via diffusion from the adipocyte.

We assume that the interstitial space immediately surrounding the adipocyte and the capillary has a radial thickness sufficiently small so that diffusion in the radial direction is nearly instantaneous, i.e., the concentration of TCE in the interstitial space depends on $(t, \theta, \phi)$ but not $r$. Therefore, we may assume that $r \approx r_{1}$ in the interstitial space. Inside the interstitial fluid, TCE diffuses across the interstitial space in both the $\theta$ and $\phi$ directions.

Based on these assumptions, the mass flux vector for the interstitial space is given by

$$
J=\left(J_{r}, J_{\theta}, J_{\phi}\right)=\left(0,-\frac{D_{I}}{r_{1} \sin \phi} \frac{\partial C_{I}}{\partial \theta},-\frac{D_{I}}{r_{1}} \frac{\partial C_{I}}{\partial \phi}\right) .
$$


The parameter $D_{I}$ is the standard diffusion coefficient with units $\mathrm{m}^{2} / \mathrm{hr}$.

Now consider an arbitrary "spherical cuboid" volume element in the interstitial space, as depicted in Figure 7. Using mass balance as before, we have

$$
\begin{aligned}
\left(\begin{array}{c}
\text { Net rate of change of TCE } \\
\text { in interstitial element }
\end{array}\right) & =\left(\begin{array}{c}
\text { Net flow rate of TCE } \\
\text { within interstitial element }
\end{array}\right) \\
& +\left(\begin{array}{c}
\text { Net flow rate of TCE } \\
\text { between interstitial } \\
\text { element and capillary }
\end{array}\right)+\left(\begin{array}{c}
\text { Net flow rate of TCE } \\
\text { between interstitial } \\
\text { element and adipocyte }
\end{array}\right) .
\end{aligned}
$$

First we consider the transport of TCE within the interstitial element only (i.e., no exchange with the other regions). Then

$$
\left(\begin{array}{c}
\text { Net flow rate of TCE } \\
\text { within interst. element }
\end{array}\right)=\iint_{\Delta S}-J \cdot \hat{n} d S,
$$

where $\hat{n}$ is the outer unit normal to the surface $\Delta S$. Dividing by the volume $\Delta V$ of the element, we obtain

$$
\left(\begin{array}{c}
\text { Net flow rate of TCE } \\
\text { within interst. element } \\
\text { per unit volume }
\end{array}\right)=\frac{1}{\Delta V} \iint_{\Delta S}-J \cdot \hat{n} d S \text {. }
$$

Using the Divergence Theorem and taking the limit as $\Delta V \rightarrow 0$ at $r=r_{1}$, we have

$$
\begin{aligned}
\left(\begin{array}{c}
\text { Net flow rate of TCE } \\
\text { within interst. element } \\
\text { per unit volume } \\
\text { at the point }\left(r_{1}, \theta, \phi\right)
\end{array}\right) & =\operatorname{Div}(-J) \\
& =-\frac{1}{r_{1}^{2}} \frac{\partial}{\partial r}\left(r_{1}^{2} J_{r}\right)-\frac{1}{r_{1} \sin \phi} \frac{\partial J_{\theta}}{\partial \theta}-\frac{1}{r_{1} \sin \phi} \frac{\partial}{\partial \phi}\left(\sin \phi J_{\phi}\right) \\
& =-\frac{1}{r_{1} \sin \phi} \frac{\partial J_{\theta}}{\partial \theta}-\frac{1}{r_{1} \sin \phi} \frac{\partial}{\partial \phi}\left(\sin \phi J_{\phi}\right) \\
& =\frac{D_{I}}{r_{1}^{2} \sin ^{2} \phi} \frac{\partial^{2} C_{I}}{\partial \theta^{2}}+\frac{D_{I}}{r_{1}^{2} \sin \phi} \frac{\partial}{\partial \phi}\left(\sin \phi \frac{\partial C_{I}}{\partial \phi}\right) .
\end{aligned}
$$

As with the capillary, we multiply by the volume and then add in the transport between the other regions to obtain

$$
\begin{aligned}
V_{I} \frac{\partial C_{I}}{\partial t} & =\frac{V_{I} D_{I}}{r_{1}^{2}}\left[\frac{1}{\sin ^{2} \phi} \frac{\partial^{2} C_{I}}{\partial \theta^{2}}+\frac{1}{\sin \phi} \frac{\partial}{\partial \phi}\left(\sin \phi \frac{\partial C_{I}}{\partial \phi}\right)\right] \\
& +\delta_{\theta_{0}}(\theta) \chi_{B}(\phi) \lambda_{I} \mu_{B I}\left(f_{B} C_{B}-f_{I} C_{I}\right)+\mu_{I A}\left(f_{A} C_{A}-f_{I} C_{I}\right)
\end{aligned}
$$

for $t \geq 0$ and $(r, \theta, \phi) \in \tilde{\Omega}_{I}$, where

$$
\tilde{\Omega}_{I}=\left\{(r, \theta, \phi): r=r_{1}, 0 \leq \theta \leq 2 \pi, 0<\phi<\pi\right\} \subset \Omega_{I}
$$

and $\chi_{B}$ is the following indicator function in $\phi$ :

$$
\chi_{B}(\phi)= \begin{cases}1 & \text { if } \phi \in\left(\varepsilon_{1}, \pi-\varepsilon_{2}\right) \\ 0 & \text { otherwise }\end{cases}
$$

This function $\chi_{B}$ is used with $\delta_{\theta_{0}}$, the Dirac delta distribution centered at $\theta=\theta_{0}$, to account for the capillary - interstitial space transport (and the capillary - adipocyte transport). 


\subsubsection{Boundary conditions}

Since we have diffusion in the interstitial space in both the $\phi$ and $\theta$ directions, we require boundary conditions in $\phi$ and $\theta$. The geometry of the spherical shell dictates periodic boundary conditions in $\theta$. That is,

$$
\begin{aligned}
C_{I}(t, \theta, \phi) & =C_{I}(t, \theta+2 \pi, \phi), \quad t \geq 0 \text { and } 0<\phi<\pi \\
\frac{\partial C_{I}}{\partial \theta}(t, \theta, \phi) & =\frac{\partial C_{I}}{\partial \theta}(t, \theta+2 \pi, \phi), \quad t \geq 0 \text { and } 0<\phi<\pi .
\end{aligned}
$$

The boundary conditions in $\phi$ are less straightforward. If we carry out separation of variables on the PDE (14) for the interstitial space, we obtain an equation in $\phi$ which is a type of singular Sturm-Liouville problem (see [16], p. 324). These problems have the form

$$
\left(p u^{\prime}\right)^{\prime}-q u+\lambda \rho u=0,
$$

where the variable coefficient $p$ vanishes at one or both of the endpoints. In our case, we have $p(\phi)=\sin \phi$, which vanishes at both $\phi=0$ and $\phi=\pi$.

The corresponding boundary value problems require a finiteness or continuity condition at the singular endpoints, taking the place of the usual type of boundary condition [16]. Here we will require the finiteness conditions

$$
\begin{aligned}
& C_{I}(t, \theta, 0)<\infty \\
& C_{I}(t, \theta, \pi)<\infty .
\end{aligned}
$$

\subsection{Derivation of equations: Adipocyte region}

In the adipocyte region, TCE enters and leaves the fat cell via diffusion across the cell membrane between the adipocyte and the interstitial space, and via diffusion between the adipocyte and capillary. We assume that diffusion of TCE in the radial direction inside the adipocyte is nearly instantaneous, so that the concentrations are uniform in $r$ for $r_{0} \leq r \leq r_{1}$. As with the interstitial space, TCE in the adipocyte diffuses across the fat cell in the $\theta$ and $\phi$ directions.

Using flux balance principles as before, we obtain the equation (taking the limit $\Delta V \rightarrow 0$ at $r=r_{0}$ )

$$
\begin{aligned}
V_{A} \frac{\partial C_{A}}{\partial t} & =\frac{V_{A} D_{A}}{r_{0}^{2}}\left[\frac{1}{\sin ^{2} \phi} \frac{\partial^{2} C_{A}}{\partial \theta^{2}}+\frac{1}{\sin \phi} \frac{\partial}{\partial \phi}\left(\sin \phi \frac{\partial C_{A}}{\partial \phi}\right)\right] \\
& +\delta_{\theta_{0}}(\theta) \chi_{B}(\phi) \lambda_{A} \mu_{B A}\left(f_{B} C_{B}-f_{A} C_{A}\right)+\mu_{I A}\left(f_{I} C_{I}-f_{A} C_{A}\right)
\end{aligned}
$$

for $t \geq 0$ and $(r, \theta, \phi) \in \tilde{\Omega}_{A}$, where

$$
\tilde{\Omega}_{A}=\left\{(r, \theta, \phi): r=r_{0}, 0 \leq \theta \leq 2 \pi, 0<\phi<\pi\right\} \subset \Omega_{A} .
$$

As before, the parameter $D_{A}$ is the diffusion coefficient with units $\mathrm{m}^{2} / \mathrm{hr}$.

\subsubsection{Boundary conditions}

The boundary conditions for the adipocyte region are similar to those for the interstitial space, and are derived using the same principles. We require periodic boundary conditions in $\theta$ and finiteness conditions in $\phi$ :

$$
\begin{aligned}
C_{A}(t, \theta, \phi) & =C_{A}(t, \theta+2 \pi, \phi), \quad t \geq 0 \text { and } 0<\phi<\pi \\
\frac{\partial C_{A}}{\partial \theta}(t, \theta, \phi) & =\frac{\partial C_{A}}{\partial \theta}(t, \theta+2 \pi, \phi), \quad t \geq 0 \text { and } 0<\phi<\pi
\end{aligned}
$$




$$
\begin{aligned}
& C_{A}(t, \theta, 0)<\infty \\
& C_{A}(t, \theta, \pi)<\infty .
\end{aligned}
$$

\subsection{Summary of model equations}

Combining the PBPK whole-body model with the adipose submodel, we obtain

$$
\begin{aligned}
& V_{B} \frac{\partial C_{B}}{\partial t}=\frac{V_{B}}{r_{2} \sin \phi} \frac{\partial}{\partial \phi}\left[\sin \phi\left(\frac{\mathcal{D}_{B}}{r_{2}} \frac{\partial C_{B}}{\partial \phi}-v C_{B}\right)\right] \\
& +\lambda_{I} \mu_{B I}\left(f_{I} C_{I}\left(\theta_{0}\right)-f_{B} C_{B}\right)+\lambda_{A} \mu_{B A}\left(f_{A} C_{A}\left(\theta_{0}\right)-f_{B} C_{B}\right) \\
& -\frac{\mathcal{D}_{B}}{r_{2}} \frac{\partial C_{B}}{\partial \phi}(t, \phi)+\left.v C_{B}(t, \phi)\right|_{\phi=\varepsilon_{1}}=\frac{Q_{c}}{1000 A_{B}} C_{a}(t) \\
& -\frac{\mathcal{D}_{B}}{r_{2}} \frac{\partial C_{B}}{\partial \phi}(t, \phi)+\left.v C_{B}(t, \phi)\right|_{\phi=\pi-\varepsilon_{2}}=\frac{Q_{c}}{1000 A_{B}} C_{v}(t) \\
& V_{I} \frac{\partial C_{I}}{\partial t}=\frac{V_{I} D_{I}}{r_{1}^{2}}\left[\frac{1}{\sin ^{2} \phi} \frac{\partial^{2} C_{I}}{\partial \theta^{2}}+\frac{1}{\sin \phi} \frac{\partial}{\partial \phi}\left(\sin \phi \frac{\partial C_{I}}{\partial \phi}\right)\right] \\
& +\delta_{\theta_{0}}(\theta) \chi_{B}(\phi) \lambda_{I} \mu_{B I}\left(f_{B} C_{B}-f_{I} C_{I}\right)+\mu_{I A}\left(f_{A} C_{A}-f_{I} C_{I}\right) \\
& C_{I}(t, \theta, \phi)=C_{I}(t, \theta+2 \pi, \phi) \\
& \frac{\partial C_{I}}{\partial \theta}(t, \theta, \phi)=\frac{\partial C_{I}}{\partial \theta}(t, \theta+2 \pi, \phi) \\
& C_{I}(t, \theta, 0)<\infty \\
& C_{I}(t, \theta, \pi)<\infty \\
& V_{A} \frac{\partial C_{A}}{\partial t}=\frac{V_{A} D_{A}}{r_{0}^{2}}\left[\frac{1}{\sin ^{2} \phi} \frac{\partial^{2} C_{A}}{\partial \theta^{2}}+\frac{1}{\sin \phi} \frac{\partial}{\partial \phi}\left(\sin \phi \frac{\partial C_{A}}{\partial \phi}\right)\right] \\
& +\delta_{\theta_{0}}(\theta) \chi_{B}(\phi) \lambda_{A} \mu_{B A}\left(f_{B} C_{B}-f_{A} C_{A}\right) \\
& +\mu_{I A}\left(f_{I} C_{I}-f_{A} C_{A}\right) \\
& C_{A}(t, \theta, \phi)=C_{A}(t, \theta+2 \pi, \phi) \\
& \frac{\partial C_{A}}{\partial \theta}(t, \theta, \phi)=\frac{\partial C_{A}}{\partial \theta}(t, \theta+2 \pi, \phi) \\
& C_{A}(t, \theta, 0)<\infty \\
& C_{A}(t, \theta, \pi)<\infty \\
& V_{v} \frac{d C_{v}}{d t}=Q_{m} C_{v m}+Q_{t} C_{v t}+Q_{f} C_{v f}+Q_{b r} C_{v b r}+Q_{l} C_{v l}+Q_{k} C_{v k}-Q_{c} C_{v} \\
& C_{a}=\frac{Q_{c} C_{v}+Q_{p} C_{c}}{Q_{c}+\frac{Q_{p}}{P_{b}}} \\
& V_{m} \frac{d C_{m}}{d t}=Q_{m}\left(C_{a}-C_{v m}\right) \\
& V_{t} \frac{d C_{t}}{d t}=Q_{t}\left(C_{a}-C_{v t}\right) \\
& V_{b r} \frac{d C_{b r}}{d t}=Q_{b r}\left(C_{a}-C_{v b r}\right) \\
& \frac{d A_{a m}}{d t}=\frac{v_{\max } C_{v l}}{k_{M}+C_{v l}}
\end{aligned}
$$




$$
\begin{aligned}
V_{l} \frac{d C_{l}}{d t} & =Q_{l}\left(C_{a}-C_{v l}\right)-\frac{v_{m a x} C_{v l}}{k_{M}+C_{v l}} \\
V_{k} \frac{d C_{k}}{d t} & =Q_{k}\left(C_{a}-C_{v k}\right) \\
\frac{d A_{i}}{d t} & =Q_{p} C_{c} \\
\frac{d A_{x}}{d t} & =Q_{p} C_{x} .
\end{aligned}
$$

\subsection{Model simulations}

We used the TCE adipose dispersion model (16) - (28) coupled with the whole-body PBPK model (29) - (38) to generate model simulations with the same input function and non-adipose parameters as in Sections 2.1 and 2.2. In these simulations we have chosen to approximate $r_{0}=r_{1}=r_{2}$. The basis for this approximation is the assumption that the radial distance between $r_{0}$ and $r_{3}$ is small compared to the radius $r_{1}$ of the adipocyte. Moreover, the radial variable $r$ occurs in our system of equations only as a coefficient, which is fixed at $r=r_{2}$ in (16), $r=r_{1}$ in (19), and $r=r_{0}$ in (24). Therefore the approximation $r_{0}=r_{1}=r_{2}$ in (16) - (28) should have little impact on the resulting solutions.

Figure 8 depicts an example simulation for the concentration of unbound TCE inside the adipocyte region versus time for several points along the "equator" $\phi=\pi / 2$, where we have chosen $\theta_{0}=\pi$. Note that these concentration profiles look similar to those for the diffusion-limited model in Figure 4.

An important difference between the diffusion-limited model simulations and the dispersion model simulations is that all of the concentration profiles in Figure 8 were generated with fixed permeability parameters $\left(\mu_{B A}=0.03, \mu_{B I}=0.02\right.$ and $\mu_{I A}=0.025$ liters/hour, $\left.\lambda_{I}=\lambda_{A}=0.5\right)$ and observations taken at varying locations, while those shown in Figure 4 were generated with varying values of the permeability coefficient $\mu$. By design, the dispersion model accounts for spatial variation in concentrations within each region of the adipose tissue, and can be used to predict various concentration profiles within the fat.

Moreover, by varying the adipose model parameters $\mathcal{F}$ and $A_{B}$, the reverse Fahraeus-Lindquist parameter and the cross-sectional area of the capillary respectively, we can control the peak concentration values inside the adipose tissue regions. This is accomplished by modifying the flux between the adipose capillary and the arterial blood compartment, as well as the flux between the adipose capillary and the venous blood compartment. The concentration profiles in Figure 8 were generated with $\mathcal{F}=0.16$ and $A_{B}=10^{-4}$, and Figure 9 depicts concentrations for $\mathcal{F}=0.08$ and $A_{B}=5 \times 10^{-5}$. Note that for a given value of $\theta$, the concentration curves are similar in shape but vary in their peak values.

Concentration profiles also can be altered significantly by changing the proportions $\lambda_{A}$ and $\lambda_{I}$ of direct and indirect transport between the capillary and the adipocyte. Figures 10 and 11 depict simulations of unbound TCE adipocyte concentrations along $\phi=\pi / 2$ for varying values of $\lambda_{A}, \lambda_{I}$. In Figure 10, the indirect transport is predominant over the direct transport $\left(\lambda_{A}=0, \lambda_{I}=1\right.$ and $\left.\lambda_{A}=0.4, \lambda_{I}=0.6\right)$, while the direct transport predominates in Figure $11\left(\lambda_{A}=0.6, \lambda_{I}=0.4\right.$ and $\left.\lambda_{A}=0.65, \lambda_{I}=0.35\right)$. As $\lambda_{A}$ increases and $\lambda_{I}$ decreases, the levels of TCE increase and the shapes of the curves change dramatically.

As seen in these example simulations, the dispersion model is able to predict concentration curves of TCE inside the adipose tissue with varying levels of accumulation and rates of decay. This suggests that this model might be successfully used with parameter estimation techniques to predict experimentally determined dynamics of TCE in adipose tissue. A paper discussing the computational efforts introduced here and the associated parameter estimation problem is currently under preparation. 


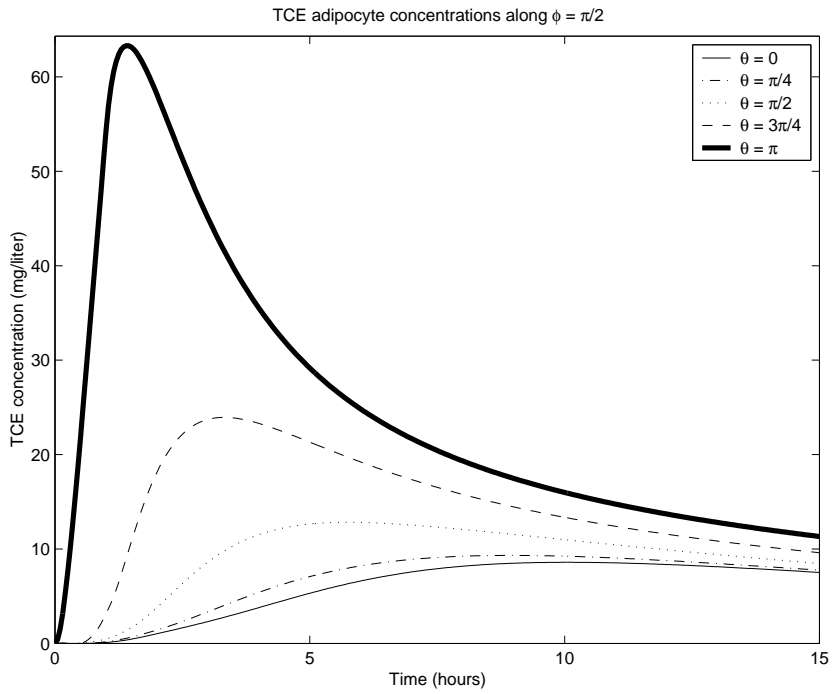

Figure 8: Model simulations: Concentrations in time of unbound TCE inside the adipocyte region of the adipose tissue at $\phi=\pi / 2$ and $\theta=0, \pi / 4, \pi / 2,3 \pi / 4$ and $\pi$, with $\mathcal{F}=0.16, A_{B}=10^{-4}, \lambda_{I}=0.5$ and $\lambda_{A}=0.5$.

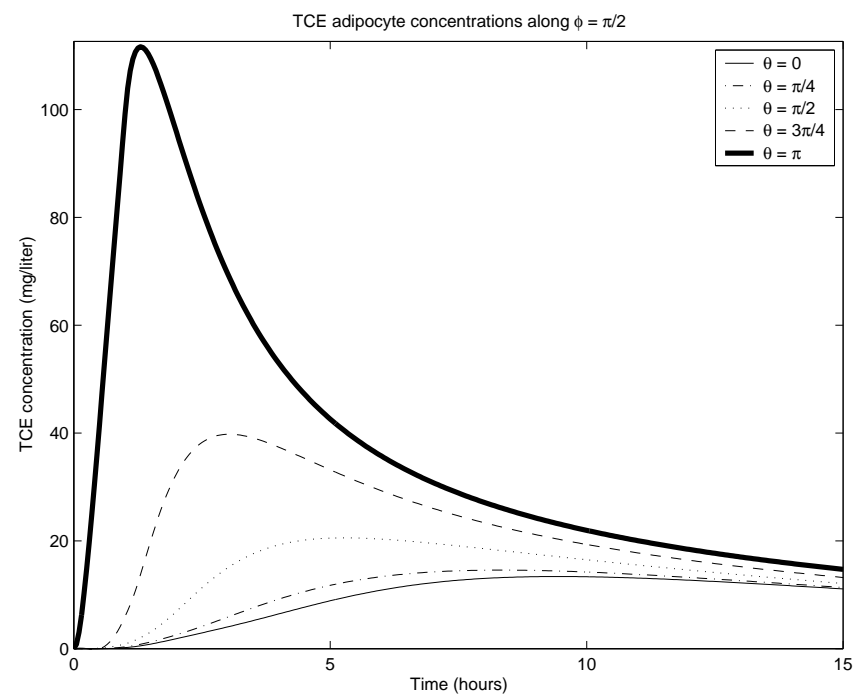

Figure 9: Model simulations: Concentrations in time of unbound TCE inside the adipocyte region of the adipose tissue at $\phi=\pi / 2$ and $\theta=0, \pi / 4, \pi / 2,3 \pi / 4$ and $\pi$, with $\mathcal{F}=0.08, A_{B}=5 \times 10^{-5}, \lambda_{I}=0.5$ and $\lambda_{A}=0.5$. 

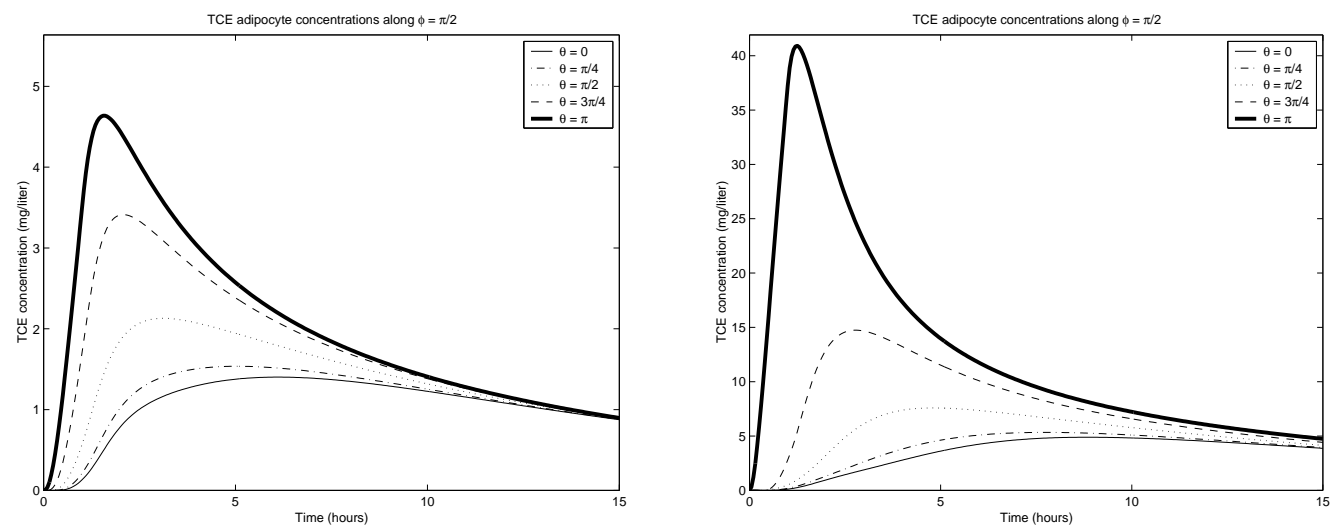

Figure 10: Model simulations: Concentrations in time of unbound TCE inside the adipocyte region of the adipose tissue at $\phi=\pi / 2$ and $\theta=0, \pi / 4, \pi / 2,3 \pi / 4$ and $\pi$, with $\mathcal{F}=0.16, A_{B}=10^{-4}$, and Left: $\lambda_{I}=1$, $\lambda_{A}=0$; Right: $\lambda_{I}=0.6, \lambda_{A}=0.4$.
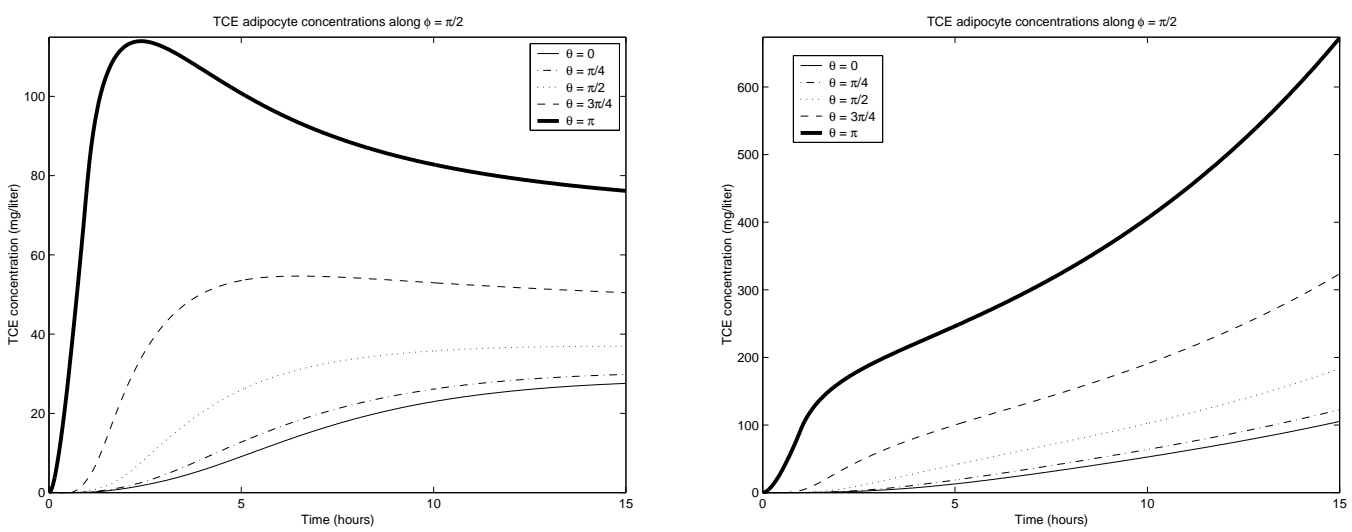

Figure 11: Model simulations: Concentrations in time of unbound TCE inside the adipocyte region of the adipose tissue at $\phi=\pi / 2$ and $\theta=0, \pi / 4, \pi / 2,3 \pi / 4$ and $\pi$, with $\mathcal{F}=0.16, A_{B}=10^{-4}$, and Left: $\lambda_{I}=0.4$, $\lambda_{A}=0.6$; Right: $\lambda_{I}=0.35, \lambda_{A}=0.65$. 


\section{Concluding remarks}

In this paper we have discussed various modeling techniques for capturing the transport behavior of trichloroethylene in the body, and specifically inside the adipose tissue. TCE is a member of a class of chemicals that is known to accumulate inside the adipose tissue, and its persistence there has a major impact on the overall kinetics of TCE inside the body. Physiologically based pharmacokinetic modeling is the standard approach for describing the disposition of compounds such as TCE in the body, and is based on a system of coupled compartmental equations that each model a specific tissue or organ.

First we implemented the standard PBPK model to simulate inhaled TCE in Long-Evans rats, using a perfusion-limited compartment for the adipose tissue. We then adapted this model by changing the fat compartment to a diffusion-limited model. The perfusion-limited model assumes that the entire tissue is in rapid equilibrium and the diffusion-limited model assumes that the intracellular space and extracellular space of a tissue are individually in rapid equilibrium. These "well-stirred" assumptions may not be adequate to capture the behavior of TCE inside the fat tissue.

Adipose is a significantly heterogeneous tissue, with widely varying physiological properties such as fat cell size, lipid distribution, blood flow rates and cell permeabilities. Each adipocyte is a relatively independent functional unit with its own blood supply, suggesting that a lipophilic compound such as TCE may have more complicated transport dynamics in the fat tissue. These dynamics are likely to be spatially varying, reflecting the spatial heterogeneities in the adipose tissue itself.

To account for these heterogeneities and more closely model the specific physiology of adipose tissue, we developed an axial dispersion type model to describe the transport of TCE inside the fat. This aggregate model uses a representative adipocyte-capillary structure to approximate the dynamics occurring in individual adipocytes throughout the tissue. The representative geometry and the model itself are based on the physiological features of adipose tissue and the expected behavior of lipophilic compounds in the body.

Model simulations were generated for various parameter sets, demonstrating that this type of model might be well-suited to predict the experimental behavior of TCE inside adipose tissue using parameter estimation techniques. Moreover, these simulations demonstrate that concentration profiles depend greatly on the choice of model parameters and on the predominant type of transport between the capillary and adipocyte regions. Based on these results and comparisons with the two standard models, it appears that accounting for the specific physiology of adipose tissue is important in developing an accurate model for the transport of TCE in the adipose tissue and the body.

\section{Acknowledgments}

This research was supported in part by the U.S. Air Force Office of Scientific Research under grants AFOSR F49620-98-1-0430, AFOSR F49620-98-1-0180, and in part by an NSF-GRT fellowship to L.K.P. under grant GER-9454175. The authors also gratefully acknowledge Dr. Hugh Barton of the National Health and Environmental Effects Research Laboratory at the U.S. Environmental Protection Agency for his insightful suggestions during early discussions on the efforts presented here.

\section{References}

[1] R. Abbas and J. Fisher. A physiologically based pharmacokinetic model for trichloroethylene and its metabolites, chloral hydrate, trichloroacetate, dichloroacetate, trichloroethanol, and trichloroethanol glucuronide in B6C3F1 mice. Toxicology and Applied Pharmacology, 147 (1997), 15-30. 
[2] N. Ahmad, W. Harsas, R.S. Marolt, M. Morton and J.K. Pollack. Total DDT and dieldrin content of human adipose-tissue. Bulletin of Environmental Contamination and Toxicology, 41 (1988), 802-808.

[3] H.T. Banks. Remarks on uncertainty of assessment and management in modeling and computation. CRSC-TR98-39, North Carolina State University, Raleigh, NC, 1998; Mathematical and Computer Modeling, 33 (2001), 39-47.

[4] H.T. Banks and K.L. Bihari. Modeling and estimating uncertainty in parameter estimation. CRSCTR99-40, North Carolina State University, Raleigh, NC, 1999; Inverse Problems, 17 (2001), 1-17.

[5] H.T. Banks, C.J. Musante and J.K. Raye. Predictions for a distributed parameter model describing the hepatic processing of 2,3,7,8-TCDD. CRSC-TR98-38, North Carolina State University, Raleigh, NC, 1998; Mathematical and Computer Modeling, 33 (2001), 49-64.

[6] H.T. Banks, C.J. Musante and H.T. Tran. A dispersion model for the hepatic uptake and elimination of 2,3,7,8-tetrachlorodibenzo-p-dioxin. Mathematical and Computer Modeling, 28 (1998), 9-29.

[7] H.A. Barton and H.J. Clewell III. Evaluating noncancer effects of trichloroethylene: dosimetry, mode of action, and risk assessment. Environmental Health Perspectives, 108 Suppl. 2 (2000), 323-334.

[8] K. Bergman. Application and results of whole-body autoradiography in distribution studies of organic solvents. CRC Critical Reviews in Toxicology, 12 (1983), 59-118.

[9] E.J. Blanchette-Mackie and R.O. Scow. Continuity of intracellular channels with extracellular space in adipose tissue and liver: demonstrated with tannic acid and lanthanum. The Anatomical Record, 203 (1982), 205-219.

[10] E.J. Blanchette-Mackie and R.O. Scow. Lipolysis and lamellar structures in white adipose tissue of young rats: lipid movement in membranes. Journal of Ultrastructure Research, 77 (1981), 295-318.

[11] E.J. Blanchette-Mackie and R.O. Scow. Membrane continuities within cells and intercellular contacts in white adipose tissue of young rats. Journal of Ultrastructure Research, 77 (1981), 277-294.

[12] H. Brauch, G. Weirich, M.A. Hornauer, S. Störkel, T. Wöhl and T. Brüning. Trichloroethylene exposure and specific somatic mutations in patients with renal cell carcinoma. Journal of the National Cancer Institute, 91 (1999), 854-861.

[13] J.V. Bruckner, B.D. Davis and J.N. Blancato. Metabolism, toxicity, and carcinogenicity of trichloroethylene. Critical Reviews in Toxicology, 20 (1989), 31-50.

[14] R.J. Bull. Mode of action of liver tumor induction by trichloroethylene and its metabolites, trichloroacetate and dichloroacetate. Environmental Health Perspectives, 108 Suppl. 2 (2000), 241260 .

[15] J.L. Byard. The toxicological significance of 2,3,7,8-tetrachlorodibenzo-p-dioxin and related compounds in adipose tissue. Journal of Toxicology and Environmental Health, 22 (1987), 381-403.

[16] R. Courant and D. Hilbert. Methods of Mathematical Physics, Vol. I. John Wiley \& Sons, New York, 1989.

[17] D. Crandall and M. DiGirolamo. Hemodynamic and metabolic correlates in adipose tissue: pathophysiologic considerations. FASEB, 4 (1990), 141-147.

[18] D. Crandall, B.M. Goldstein, F. Huggins and P. Cervoni. Adipocyte blood flow: influence of age, anatomic location, and dietary manipulation. American Journal of Physiology, 247 (1984), R46-R51. 
[19] D. Crandall, G.J. Hausman and J.G. Kral. A review of the microcirculation of adipose tissue: anatomic, metabolic, and angiogenic perspectives. Microcirculation, 4 (1997), 211-232.

[20] I.W.F. Davidson and R.P. Beliles. Consideration of the target organ toxicity of trichloroethylene in terms of metabolite toxicity and pharmacokinetics. Drug Metabolism Reviews, 23 (1991), 493-599.

[21] C. Di Franceso and M.H. Bickel. Uptake in vitro of lipophilic model compounds into adipose tissue preparations and lipids. Biochemical Pharmacology, 34 (1985), 3683-3688.

[22] M.V. Evans, W.K. Boyes, P.J. Bushnell, J.H. Raymer and J.E. Simmons. A Physiologically Based Pharmacokinetic Model for Trichloroethylene (TCE) in Long-Evans Rats. Personal communication, 1999.

[23] R.W. Fahien. Fundamentals of Transport Phenomena. McGraw-Hill, New York, 1983.

[24] J.W. Fisher, M.L. Gargas, B.C. Allen and M.E. Andersen. Physiologically based pharmacokinetic modeling with trichloroethylene and its metabolite, trichloroacetic acid, in the rat and mouse. Toxicology and Applied Pharmacology, 109 (1991), 183-195.

[25] J.W. Fisher, D. Mahle and R. Abbas. A human physiologically based pharmacokinetic model for trichloroethylene and its metabolites, trichloroacetic acid and free trichloroethanol. Toxicology and Applied Pharmacology, 152 (1998), 339-59.

[26] R.L. Fournier. Basic Transport Phenomena in Biomedical Engineering. Taylor \& Francis, Philadelphia, 1999.

[27] Y.C. Fung. Biomechanics: Mechanical Properties of Living Tissues. Springer-Verlag, New York, 1981.

[28] A.R. Goeptar, J.N.M. Commandeur, B. van Ommen, P.J. van Bladeren and N.P.E. Vermeulen. Metabolism and kinetics of trichloroethylene in relation to toxicity and carcinogenicity. Relevance of the mercapturic acid pathway. Chemical Research in Toxicology, 8 (1995), 3-21.

[29] T. Green. Pulmonary toxicity and carcinogenicity of trichloroethylene: species differences and modes of action. Environmental Health Perspectives, 108 Suppl. 2 (2000), 261-264.

[30] M.S. Greenberg, G.A. Burton and J.W. Fisher. Physiologically based pharmacokinetic modeling of inhaled trichloroethylene and its oxidative metabolites in B6C3F1 mice. Toxicology and Applied Pharmacology, 154 (1999), 264-78.

[31] A.C. Guyton. Textbook of Medical Physiology. W.B. Saunders Company, Philadelphia, 1991.

[32] G.J. Hausman. The comparative anatomy of adipose tissue. In New Perspectives in Adipose Tissue: Structure, Function and Development. Butterworths, London, 1985.

[33] L.H. Lash, J.W. Fisher, J.C. Lipscomb and J.C. Parker. Metabolism of trichloroethylene. Environmental Health Perspectives, 108 Suppl. 2 (2000), 177-200.

[34] L.H. Lash, J.C. Parker and C.S. Scott. Modes of action of trichloroethylene for kidney tumorigenesis. Environmental Health Perspectives, 108 Suppl. 2 (2000), 225-240.

[35] O. Levenspiel. Chemical Reaction Engineering, 3rd Ed. John Wiley and Sons, New York, 1999.

[36] R.A. Lordo, K.T. Dinh and J.G. Schwemberger. Semivolatile organic compounds in adipose tissue: estimated averages for the US population and selected subpopulations. American Journal of Public Health, 86 (1996), 1253-1259.

[37] H.A. MacQueen, V. Waights and C.M. Pond. Vascularization in adipose depots surrounding immunestimulated lymph nodes. Journal of Anatomy, 194 (1999), 33-38. 
[38] M.A. Medinsky and C.D. Klaassen. Toxicokinetics. In Casarett and Doull's Toxicology: the Basic Science of Poisons, 5th Ed. McGraw-Hill, Health Professions Division, New York, 1996.

[39] S. Minder, W.A. Daniel, J. Clausen and M.H. Bickel. Adipose-tissue storage of drugs as a function of binding competition - in-vitro studies with distribution dialysis. Journal of Pharmacy and Pharmacology, 46 (1994), 313-315.

[40] G.M. Pastino, W.Y. Yap and M. Carroquino. Human variability and susceptibility to trichloroethylene. Environmental Health Perspectives, 108 Suppl. 2 (2000), 201-215.

[41] M.S. Roberts and M. Rowland. A dispersion model of hepatic elimination: 1. Formulation of the model and bolus considerations. Journal of Pharmacokinetics and Biopharmaceutics, 14 (1986), 227260 .

[42] S. Rosell and E. Belfrage. Blood circulation in adipose tissue. Physiological Reviews, 59 (1979), 1078-1104.

[43] A.M. Saillenfait, I. Langonne and J.P. Sabate. Developmental toxicity of trichloroethylene, tetrachloroethylene and four of their metabolites in rat whole embryo culture. Archives of Toxicology, $\mathbf{7 0}$ (1995), 71-82.

[44] R.O. Scow, E.J. Blanchette-Mackie and L.C. Smith. Transport of lipid across capillary endothelium. Federation Proceedings, 39 (1980), 2610-2617.

[45] T.M. Simon, F. Reitich, M.R. Jolly, K. Ito, and H.T. Banks. The effective magnetic properties of magnetorheological fluids. Mathematical and Computer Modelling, 33 (2001), 273-284.

[46] T.M. Simon, F. Reitich, M.R. Jolly, K. Ito, and H.T. Banks. Estimation of the effective permeability in magnetorheological fluids. CRSC-TR98-35, North Carolina State University, Raleigh, NC, 1998; Journal of Intelligent Material Systems and Structures, 10 (1999), 872-879.

[47] B.G. Slavin. The morphology of adipose tissue. In New Perspectives in Adipose Tissue: Structure, Function and Development. Butterworths, London, 1985.

[48] R.D. Stenner, J.L. Merdink, J.W. Fisher and R.J. Bull. Physiologically-based pharmacokinetic model for trichloroethylene considering enterohepatic recirculation of major metabolites. Risk Analysis, 18 (1998), 261-269.

[49] I.R. Telford and C.F. Bridgman. Introduction to Functional Histology. HarperCollins College Publishers, New York, 1995.

[50] United Nations Environment Programme, International Labour Organisation, and World Health Organization. Trichloroethylene. World Health Organization, Geneva, 1985.

[51] D.B. West, W.A. Prinz, A.A. Francendese and M.R.C. Greenwood. Adipocyte blood flow is decreased in obese Zucker rats. American Journal of Physiology, 253 (1987), R228-R233. 